\title{
Uma ferramenta gráfica para suporte à atividade docente no Moodle
}

\author{
Felipe Dias Costa Zielinski, IFRS, zielinski.felipe@ gmail.com* \\ Marcelo Augusto Rauh Schmitt - IFRS - marcelo.schmitt@poa.ifrs.edu.br ${ }^{\dagger}$
}

\begin{abstract}
Resumo. Este artigo tem como objetivo apresentar uma ferramenta gráfica para suporte à atividade docente no ambiente virtual de aprendizagem Moodle. São apresentadas as bases teóricas do trabalho, a metodologia e as tecnologias empregadas, bem como os resultados obtidos. A partir dos estudos realizados, foi desenvolvido um plugin para o ambiente virtual de aprendizagem Moodle. O módulo encontra-se operacional e está disponível para instalação no diretório de extensões desta ferramenta. A partir dele, é possível ao docente visualizar de maneira ágil se determinado aluno está acessando os conteúdos disponibilizados, está participando das atividades, ou mesmo se está frequentando o ambiente. A apresentação gráfica permite a rápida identificação de situações de risco e a intervenção pedagógica através da comunicação com o discente.
\end{abstract}

Palavras-chave: Learning Analytics, Ambiente Virtual de Aprendizagem

\section{A graphic tool for supporting teaching in Moodle}

Abstract. This paper presents a graphical tool that supports teacher's activity in Moodle Learning Management System. We introduce he theoretical foundations of the work, the used methodology and technologies, and the software resulted from the research. Based on the investigations carried on, we developed a Moodle plugin. The module is operational and it is available for downloading at the learning management system extension directory. The tool allows teachers to easily visualze whether a student is accessing any content, participating in the activities, or even if he is consulting the course. The information graphic format and the integrated email tool allow for quick identification of risky situations and pedagogical intervention.

Keywords: Learning Analytics, Learning Management System

\section{Introdução}

A educação a distância (EAD) teve início no Brasil em 1904, quando era possível participar de cursos de escolas norte-americanas por correspondência. Desde então, novas tecnologias foram sendo utilizadas. Em 1937, foi criado o Serviço de Radiodifusão Educativa (MEC), e o Programa Nacional de Teleducação (PRONTEL) surgiu em 1972. Atualmente, a Internet é tecnologia fundamental na implantação de projetos federais de incentivo à EAD, tais como os programa Universidade Aberta do Brasil (UAB) e Rede E-TEC Brasil. A importância da educação a distância no Brasil pode ser atestada pela criação da Associação Brasileira dos Estudantes de Educação a Distância (ABE-EAD), em 2008 (LIMA, 2014).

Esta modalidade de ensino traz novos desafios aos docentes. A distância entre docentes e discentes, por óbvio inerente ao processo, dificulta o acompanhamento do

\footnotetext{
*Bolsista BICTES (Bolsa de Iniciação Científica / Ensino Superior) do IFRS.

${ }^{\dagger}$ Agradecemos o apoio financeiro do $\mathrm{CNPq}$ à pesquisa realizada.
} 
desenvolvimento dos alunos. A comunicação face-a-face permite mais facilmente avaliar a motivação, a participação e o crescimento discentes. O próprio Ministério da Educação identifica tal dificuldade em (MEC, 2014b).

Cabe ao professor, seja em cursos presenciais ou a distância, avaliar constantemente os seus alunos e criar novas estratégias de ensino. É preciso identificar quais alunos necessitam abordagens específicas, quais não estão acompanhando o desenvolvimento da disciplina ou curso, ou mesmo quais alunos estão em vias de abandonar os estudos. Em cursos presenciais isto é feito no contato diário ou semanal com o aluno e na aplicação de avaliações formativas. Em um curso na modalidade EAD, ocorrem duas dificuldades básicas: a falta da interação face-a-face e a quantidade de alunos envolvidos. Há necessidade, portanto, de fornecer um suporte adequado para que o docente possa identificar a forma como o aluno está participando da disciplina.

Nos cursos a distância desenvolvidos através das modernas tecnologias de informação e comunicação, o Ambiente Virtual de Aprendizagem (AVA) tem um papel preponderante. De forma geral, é no AVA que o aluno busca os conteúdos da disciplina, realiza as tarefas, interage com os colegas, com os tutores e com os professores. Quando adequadamente configurados, estes ambientes armazenarão as ações dos alunos e conterão informações importantes para a tomada de decisão por parte dos docentes. Afinal, pelos registros guardados pelos AVAs, é possível obter informações a respeito do nível de participação do aluno e da sua evolução na disciplina.

O presente trabalho busca identificar formas de fornecer tal subsídio para que o professor, mesmo diante das dificuldades impostas pela EAD, possa tomar decisões pedagógicas adequadas. Desenvolveu-se ferramenta de computação que, integrada ao AVA, dá suporte ao trabalho docente e facilita a ação sobre os diferentes perfis de alunos.

A seção dois discute desafios inerentes à educação a distância no que diz respeito ao acompanhamento do aluno. A seção três apresenta a área de pesquisa denominada Learning Analytics (LA). Tal contextualização é importante porque a pesquisa claramente está enquadrada neste campo de investigação relativamente novo dentro das tecnologias educacionais. A seção quatro trata da oportunidade do uso de gráficos neste tipo de software. Finalmente, a seção cinco apresenta a metodologia e as tecnologias empregadas, assim como as ferramentas já desenvolvidas no âmbito deste trabalho.

\section{Desafio da educação a distância}

Segundo MEC (2014a), uma modalidade de ensino pode ser considerada EAD na medida em que professores e alunos estejam separados física ou temporalmente, sendo necessário o uso de tecnologias de informação e comunicação. Esta modalidade possui uma legislação específica, podendo ser implantada da educação básica até o nível superior. Por não necessitar de um elo físico ou temporal entre o aluno e o professor, a EAD apresenta vantagens sobre outros métodos: flexibilidade, permitindo ao aluno decidir qual será o horário ideal para realizar seus estudos; autonomia, pela iniciativa dos estudos ter que partir do aluno; e variedade de materiais, pela possibilidade de utilização de elementos multimídia, como vídeo, áudio, animações gráficas, documentos de diferentes formatos, imagens, slides e outros.

Uma vez que não há como igualar a experiência educacional presencial com aquela que ocorre na EAD, novas questões surgem para a manutenção da qualidade destes cursos. Segundo MEC (2014b), pode ser necessário na introdução do curso um 
módulo introdutório que desenvolva habilidades básicas referentes à tecnologia utilizada e/ou ao conteúdo programático, promovendo um nivelamento inicial comum a todos estudantes. De acordo com Konrath (2009), os professores e tutores são os responsáveis pela mediação pedagógica, tendo entre suas atribuições tarefas como: organizar os materiais no ambiente virtual de aprendizagem, orientar os alunos, responder as dúvidas operacionais e sobre o conteúdo, comentar as produções e interagir nas ferramentas de discussão, acompanhar e avaliar os alunos. Além disso o ambiente da EAD exige que o aluno aja de maneira ativa, pois não é suficiente a visualização de materiais para que ocorra a construção de conhecimento, é necessário que o aluno interaja com o ambiente como um todo, seja através de contribuições com colegas, interagindo com ferramentas ou participando de discussões. Isso exige que o aluno tenha, principalmente, autonomia e disciplina para que mantenha o mesmo nível de dedicação durante o curso. Assim sendo, são exigidas novas competências e habilidades que são fundamentais no EAD mas que também acabam sendo úteis no ensino presencial. O EAD necessita da interação desse atores para que suas funções conjuntas formem uma rede interativa cujo eixo central seja a construção de conhecimento.

Segundo Silva (2012), no EAD é imprescindível a criação de métodos e ferramentas que capacitem o professor a realizar uma observação detalhada dos alunos. Isso faz parte, inclusive, do método de avaliação e do acompanhamento formativo dos alunos. Cabe ao AVA servir de intermediário entre professores e alunos também dando dinâmica às aulas teóricas através de videoaulas, exercícios, chats e fóruns. Entre os AVAs existentes, há sistemas pagos como BlackBoard e outros gratuitos de código-aberto como Moodle, que é o sistema para o qual foi desenvolvida a ferramenta aqui apresentada. De acordo com Moodle.org (2014), o AVA Moodle é capaz de armazenar dados como:

- Nível de ensino: um evento ou ação realizada por um professor (geralmente) que afeta a experiência de aprendizagem dos alunos; por exemplo, a classificação de um aluno ou a adição de um módulo para o curso.

- Nível de participantes: um evento ou ação que pode estar relacionado à experiência de aprendizagem de um usuário; por exemplo, um estudante postando um fórum ou enviando uma atribuição.

\section{Learning Analytics}

\subsection{O que é Learning Analytics}

Este trabalho enquadra-se em um campo de pesquisa denominado Learning Analytics. Este termo ainda não apresenta uma tradução consagrada na literatura nacional. Segundo Dimopoulos et al. (2013), o LA é um campo de pesquisa emergente com muitas ferramentas implementadas, visando fornecer aos educadores os recursos para que eles reconheçam e avaliem seu processo educativo. O LA também pode ser definido como o uso de dados inteligentes (produzidos pelos alunos) e de modelos de análise com o objetivo de descobrir informações e conexões sociais, e a previsão e aconselhamento sobre o aprendizado (LONG; SIEMENS, 2011). De acordo com Retalis (2006), LA pretende principalmente ajudar professores e alunos a agirem com base na avaliação de dados educacionais.

Ainda segundo Shun (2012), o LA pode ser definido como um termo que originou-se da junção de técnicas de análise de dados da área de Business Inteligence (BI) com a experiência do aprendizado. Este mesmo autor identifica 3 níveis na sua aplicação: 
- Micro - Neste nível, o acompanhamento e análise de dados se dá individualmente (ou em pequenos grupos). A ideia é fornecer informações sobre o desenvolvimento do aluno ou de um grupo de alunos. Esta gama de dados é de suma importância pois é capaz de prover respaldo sobre possíveis problemas de desempenho e permite fornecer maior detalhes sobre o aluno através de mecanismos clique-a-clique, geolocalização, empréstimos na biblioteca e/ou visualizações de materiais;

- Meso - Este nível de aplicação relaciona-se com questões institucionais. Aqui percebe-se mais o papel da BI. É possível obter-se informações compartilhadas entre diferentes setores da instituição de ensino, a fim de que se tomem decisões críticas com maior precisão, visando sempre a otimizar processos e criar um "ambiente de análise acadêmica";

- Macro - Por último, no nível macro são englobados dados de várias instituições com toda vida acadêmica do aluno, juntando informações dos níveis micro e meso importantes para que seja possível definirem recursos e novas políticas para obterem-se melhores resultados em níveis municipais, estaduais ou até mesmo nacionais.

Este trabalho concentra-se no nível micro definido por Shun (2012), buscando apresentar dados individuais de alunos, ou mesmo agrupados, com a intenção de prover informações aos professores com vistas ao desenvolvimento discente.

Assim como Shun (2012) definiu níveis de aplicação para LA, Pardo (2014) sugeriu que a análise de dados educacionais ocorrem em cinco etapas distintas: captura, relatório, prevenção, ato e refino. Decisões tomadas nos estágios iniciais têm forte influência sobre os passos seguintes. No estágio de captura, são tomadas decisões e adotadas técnicas para certificar-se que os dados necessários nos próximos passos serão armazenados. Como essas informações podem provir de diversas fontes, não necessariamente estarão prontas para leitura. Na parte de relatório, é assumido que os dados obtidos nos passos anteriores são processados por métodos que vão desde a simples visualização até algoritmos mais complexos que resumem e combinam dados. Já a prevenção é um passo que necessita da atuação dos responsáveis pelo sistema (professores, administradores escolares, políticos). Neste estágio, as aplicações fornecem as respostas para questões previamente formuladas como, por exemplo, a probabilidade de abandono do curso (Romero et al.2013). O ato talvez seja o passo mais sofisticado, porque consiste em gerar ações que mudem aspectos da atividade de aprendizado, podendo executar ações que deem maior suporte a alunos que estão perdendo engajamento. Como último estágio, o refino é responsável por manter a supervisão e a revisão dos passos anteriores, procurando garantir que os dados sejam coletados e aplicados sobre o indivíduo correto, na condição correta e com máximo impacto.

\subsection{Diferença entre Learning Analytics e Educational Data Mining}

Conforme Romero (2010), Educational Data Mining (EDM) é a aplicação de técnicas de Data Mining (DM) na área da educação, desenvolvendo métodos que exploram dados em ambientes educacionais para entender o comportamento dos alunos e padrões de aprendizado. Com o aumento de softwares como instrumentos educacionais e bancos de dados estatais sobre os estudantes, foram criados grandes repositórios de dados capazes de refletir o aprendizado do aluno. Além disso, há uma imensa quantidade de informações providas por sistemas de suporte a EAD. Todas essas informações são a fonte de dados do EDM. Apesar de EDM aparentar ser um sinônimo de LA, há algumas diferenças básicas que acarretam a separação dos grupos de pesquisa. Essas diferenças dizem respeito, 
basicamente, ao papel dos sistemas especialistas na tomada de decisão. Em LA, o princípio fundamental é fornecer informações para que professores ou outros profissionais envolvidos tomem decisões; em EDM, procuram-se criar mecanismos automáticos de ação.

"Ao contrário de Educational Data Mining, Learning Analytics não aborda, geralmente, o desenvolvimento de novos métodos computacionais para a análise de dados; aborda sim a aplicação de métodos e modelos conhecidos para responder a questões importantes que afetam a aprendizagem do estudante e sistemas de aprendizagem organizativos... As instituições de ensino superior têm mostrado maior interesse em Learning Analytics, uma vez que elas têm sido alertadas para maior transparência e maior escrutínio das suas práticas de recrutamento e retenção.”(FARIA, 2014)

Os campos de investigação denominados LA e EDM podem ter finalidades semelhantes, porém os métodos adotados para que cada um chegue aos seus objetivos têm algumas diferenças, como pode ser visto na Tabela 1.

Tabela 1. Diferenças entre Learning Analytics e Educational Data Mining

\begin{tabular}{|c|c|c|}
\hline & Learning Analytics & Educational Data Mining \\
\hline Tipo de descoberta & $\begin{array}{l}\text { O julgamento humano é } \\
\text { a chave e a descoberta } \\
\text { automatizada ér uma } \\
\text { ferramenta para alcançar } \\
\text { este objetivo. }\end{array}$ & $\begin{array}{l}\text { O julgamento humano é uma } \\
\text { ferramenta para se chegar a } \\
\text { descoberta automatizada }\end{array}$ \\
\hline Visão reducionista ou holística & $\begin{array}{l}\text { Maior ênfase na compreensão } \\
\text { do sistema geral, em toda a } \\
\text { sua complexidade. }\end{array}$ & $\begin{array}{l}\text { Maior ênfase na redução do } \\
\text { sistema para componentes } \\
\mathrm{e} \text { análise das relações } \\
\text { individuais entre eles. }\end{array}$ \\
\hline Origens & $\begin{array}{l}\text { Origens mais fortes em } \\
\text { web semântica, "currículo } \\
\text { inteligente", previsão de } \\
\text { resultados e intervenções } \\
\text { sistêmicas. }\end{array}$ & $\begin{array}{l}\text { Fortes origens em software } \\
\text { educacional e modelagem do } \\
\text { estudante, com ênfase na } \\
\text { previsão de resultados do } \\
\text { curso. }\end{array}$ \\
\hline Adaptação e Personalização & $\begin{array}{l}\text { Maior enfoque em fornecer } \\
\text { informações e poder a } \\
\text { instrutores e alunos. }\end{array}$ & $\begin{array}{l}\text { Maior enfoque na adaptação } \\
\text { automática sem intervenção } \\
\text { humana. }\end{array}$ \\
\hline Técnicas e Métodos & $\begin{array}{l}\text { Análise social da rede, } \\
\text { análise de sentimento, análise } \\
\text { de influência, a análise do } \\
\text { discurso, a previsão successo, } \\
\text { a análise de concepção, } \\
\text { modelos de sensemaking. }\end{array}$ & $\begin{array}{lr}\text { Classificação, } & \text { clustering, } \\
\text { modelagem } & \text { Bayesiana, } \\
\text { mineração } & \text { de } \\
\text { relacionamento, } & \text { descoberta } \\
\text { com modelos, visualização. }\end{array}$ \\
\hline
\end{tabular}

(SIEMENS; BAKER, 2012)

\section{O uso de gráficos em LA}

Os diferentes tipos de representação gráfica são uma importante ferramenta para análise e interpretação de um determinado conjunto de dados. Sua importância está ligada à facilidade e à rapidez na interpretação das diferentes informações. Grandes instituições financeiras, por exemplo, utilizam gráficos para informar lucros e prejuízos fornecendo aos interessados, além de uma informação de fácil compreensão, um panorama geral dessse conjunto de dados.

AVAs como o Moodle apresentam informações para que os professores possam perceber o comportamento do aluno em um curso a distância. No entanto, a obtenção dessas informações não é trivial. Algumas estão disponíveis apenas nos registros dos 
sistema e exigem a leitura de algo que não é natural para o professor e sim para um profissional de informática: linhas de registros. Outras são apresentadas de forma mais amigável mas sem o nível de agregação necessário para uma compreensão facilitada de dados volumosos.

De acordo com Brooks, Greer e Gutwin (2014), é importante prover ao professor uma compreensão estatística e gráfica das relações entre os diversos grupos de alunos. Para os autores, os dados devem ser resumidos e correlacionados a fim de facilitar a atividade docente. Já para Mazza e Dimitrova (2007), ao visualizar gráficos, o professor pode formar modelos mentais do que está ocorrendo em sua sala de aula. Os autores ressaltam que é possível aumentar a efetividade dos AVAs através da integração de técnicas de visualização da informação. Em pesquisa realizada por Ali et al. (2012), constatou-se que os educadores valorizam a combinação de informações textuais e gráficas. Também percebeu-se que a utilização de gráficos é uma forma de permitir a compreensão de grande quantidade de dados.

O uso de gráficos para transmitir informações aos professores pode ser considerado o principal impacto da BI (Bussiness Inteligence) nos AVAs. Tentar apresentar informações que já estão disponíveis, porém de difícil compreensão, em um formato que os usuários não-técnicos possam interpretar facilmente, seja através de uma variedade de gráficos, tabelas e outras visualizações e relatórios personalizados projetados para o consumo de educadores, administradores e analistas de dados é fundamental (SHUN, 2012).

Uma ferramenta de LA, portanto, não deve limitar-se a recuperar dados dos registros de um sistema computacional e apresentá-los para o professor. É preciso organizá-los de forma a facilitar a cognição do docente que precisa tomar decisões pedagógicas com segurança.

\section{Metodologia, tecnologias e produto desenvolvido}

Levando-se em conta o papel relevante que AVAs desempenham na educação a distância, ou mesmo em modelos educacionais híbridos, a necessidade de prover o docente com informações sobre a caminhada do aluno em determinada disciplina, e as vantagens de organizar informações em um formato de mais fácil compreensão, decidiu-se investigar possíveis contribuições para o problema. A metodologia empregada é denominada Design Based Research (DBR) (BARAB; SQUIRE, 2004) (WANG; HANNAFIN, 2005), adequada para pesquisas aplicadas na área de tecnologia educacional. A ideia norteadora da metodologia é a criação de uma intervenção pedagógica com a participação dos usuários. As sucessivas interações e refinamentos procuram aperfeiçoar a proposta e o produto resultante dela.

A partir do levantamento das ferramentas mais utilizadas no AVA do Campus Porto Alegre do Instituto Federal do Rio Grande do Sul (IFRS), foram definidas três interfaces de suporte para tomada de decisão do professor. Estas intervenções estão sendo testadas e aprimoradas em dois cursos técnicos a distância do mesmo campus: Administração e Redes de Computadores. Os testes são realizados com a participação de docentes para refinamento e melhoria das interfaces, além da definição de novas configurações.

A fim de construírem-se as interfaces, têm sido empregadas as seguintes tecnologias: Moodle, PHP, jQuery, highCharts.js, HTML e CSS. O Moodle foi utilizado 
por tratar-se do AVA empregado no IFRS e também na Universidade Federal do Rio Grande do SUL (UFRGS), parceiros no projeto. Foi desenvolvido um plugin para este ambiente virtual de aprendizagem, disponível tanto no diretório de extensões do Moodle $^{1}$, quanto no repositório Github ${ }^{2}$. Este plugin é compatível com as versões 2.2 e superiores do Moodle. A fim de produzir gráficos, utilizou-se a biblioteca denominada highCharts.js ${ }^{3}$. A interatividade da ferramenta fica por conta das linguagens utilizadas.

Até o presente momento, foram definidos três tipos de gráficos:

- Visualização de conteúdos acessados

- Visualização de submissões

- Visualização de participação no ambiente

A visualização de conteúdos acessados dá-se através do gráfico apresentado na Figura 1. Cada conteúdo é disposto em uma linha do lado esquerdo do gráfico, de acordo com o tópico em que se encontra. Os tópicos são identificados do lado direito do gráfico e os acessos são explicitados através de barras e números. A barra verde indica a quantidade de alunos que acessou determinado conteúdo; a vermelha, a quantidade que não acessou. É possível realçar determinada parte do gráfico (zoom).

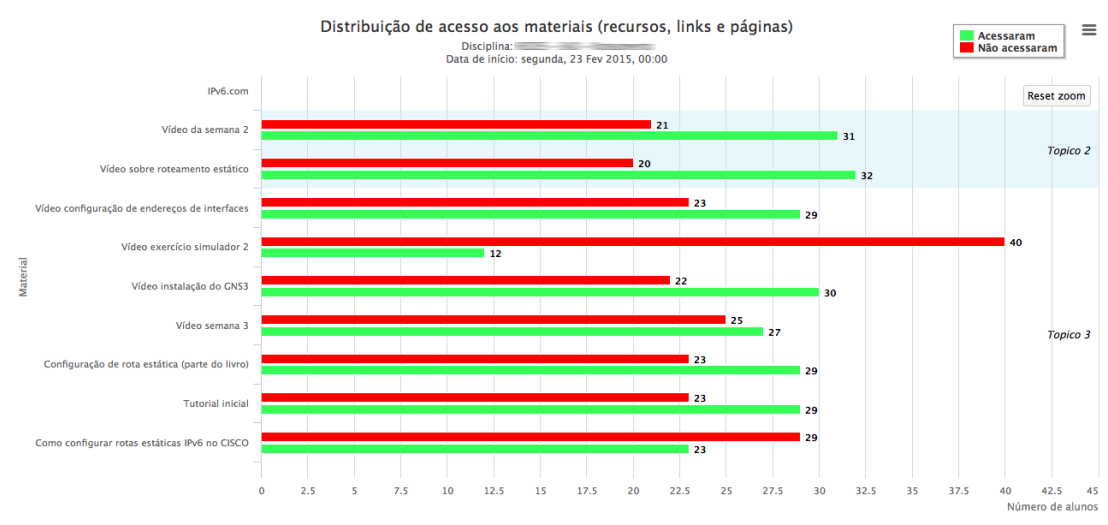

Figura 1. Distribuição de acesso aos materiais

A apresentação nesse formato permite que o docente verifique rapidamente a proporção de acessos, os conteúdos com maior ou menor acesso, e mesmo a evolução ao longo do tempo se considerarmos que os tópicos são sequenciais. Ao clicar sobre uma das barras, o docente tem acesso a um formulário para envio de mensagem eletrônica ao conjunto de alunos ali representados. Por exemplo, ao clicar sobre a barra vermelha de determinado conteúdo, é possível enviar mensagem a todos alunos que não o acessaram.

A visualização das submissões é realizada com um gráfico com três barras verticais para cada atividade que exige entrega de algum tipo de reposta (Figura 2). As barras identificam a quantidade de alunos que realizaram submissões no tempo correto, realizaram submissões atrasadas, ou não realizaram submissões. Para enviar uma mensagem a determinado grupo de alunos, basta clicar sobre a barra que identifica tal grupo. Além das barras, o gráfico também apresenta duas linhas que mostram a evolução de dois parâmetros relacionados à submissão de trabalhos: proporção de pontualidade e proporção de entrega. Desta forma, o professor pode, rapidamente, saber se em uma

\footnotetext{
${ }^{1}$ https://moodle.org/plugins/view/block_analytics_graphs

${ }^{2}$ https://github.com/marceloschmitt/moodle-block_analytics_graphs

${ }^{3}$ http://www.highcharts.com
} 
disciplina, com o passar do tempo, os alunos estão evoluindo no cumprimento dos prazos ou na entrega das tarefas.

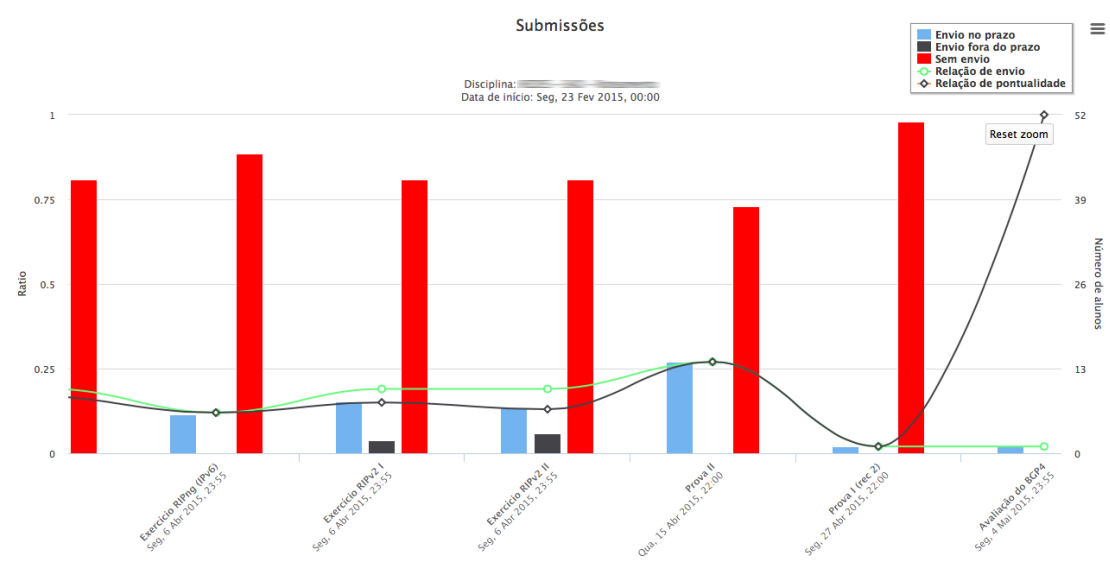

Figura 2. Submissões de trabalhos

A fim de tornar a implementação reutilizável para diferentes tipos de módulos do Moodle que utilizam o conceito de submissão, definiu-se uma classe que é reaproveitada em diferentes casos. A partir desta classe foram realizados gráficos para visualização de tarefas (assignment), questionários (quizz) e exercícios hotpotatoes.

Por último, a visualização da participação no ambiente ocorre em um relatório que congrega dados numéricos e gráficos (Figura 3). Os alunos são ordenados por ordem alfabética e é possível enviar mensagem para um aluno individualmente clicando-se sobre o seu nome. Também é possível enviar mensagem para o conjunto de alunos que nunca tenham acessado a disciplina.

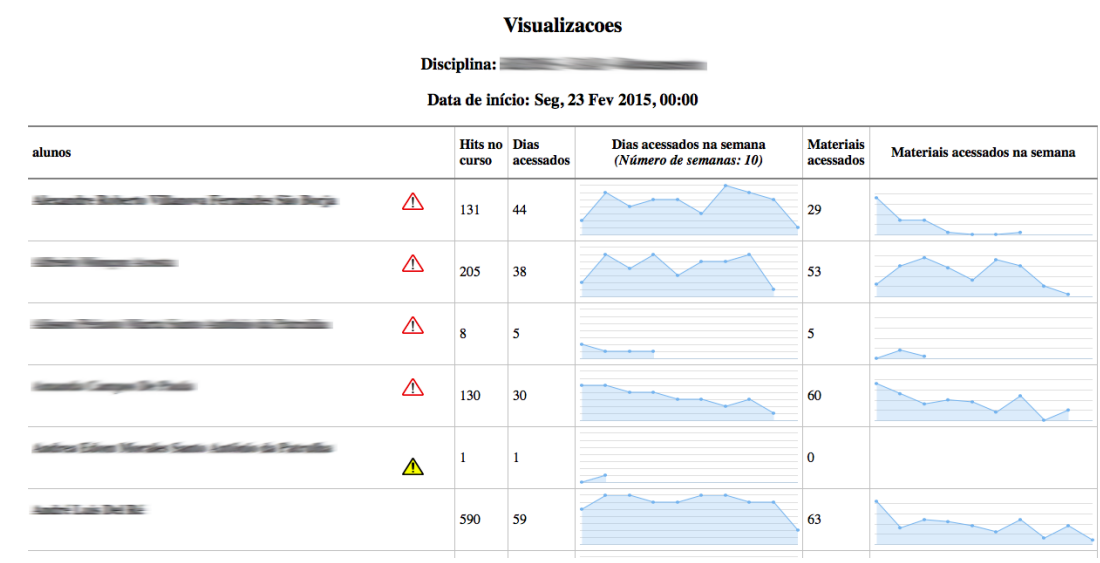

Figura 3. Visualizações no curso

O relatório permite saber o número de hits na disciplina, o número de dias acessados e o número de materiais distintos visualizados. Os gráficos fornecem ao professor informações sobre o comportamento do aluno ao longo do tempo: quantos dias por semana o aluno entra no ambiente e quantos materiais ele acessa também por semana. O professor pode, assim, identificar rapidamente discentes que estejam diminuindo seu ritmo ou que tenham um nível de participação diferente dos colegas.

Por tratar-se de uma pesquisa do tipo DBR, o produto desenvolvido está sendo utilizado como uma intervenção pedagógica no IFRS e no Centro Interdisciplinar de 
Novas Tecnologias na Educação da UFRGS (CINTED). Os professores que o utilizam são parte fundamental do processo investigativo. A partir do uso inicial do produto, identificaram-se algumas modificações que estão sendo acrescidas à nova versão do software.

Constatou-se ser necessária a organização dos gráficos por grupos de alunos já que em muitos cursos EAD no Brasil, os polos são organizados utilizando-se grupos dentro de disciplinas. A apresentação de todos os alunos impede que tutores de polos possam particularizar suas mensagens e também dificulta a visualização da cada turma individualmente. Verificou-se que as mensagens enviadas pelos professores aos alunos deve ser replicada para todos os outros professores ou tutores. Isto evita que ocorra repetição e permite que todo o corpo docente tenha pleno conhecimento das intervenções que estão sendo realizadas. Finalmente, percebeu-se que para um compreensão melhor das intervenções realizadas ao longo do tempo é necessário que as mensagens enviadas estejam disponíveis de forma organizada. Assim, um professor pode verificar que comunicações já estabeleceu com os diversos alunos.

Acredita-se que as sugestões trazidas pelos usuários enriquecem a proposta e permitem que o produto resultante da pesquisa seja valioso nas atividades docentes. Tal premissa será verificada a partir de estudo mais detalhado do uso da ferramenta em diferentes cursos.

\section{Conclusões}

O plugin desenvolvido preenche uma lacuna encontrada em ambientes virtuais de aprendizagem como o Moodle: a falta de informações claras que permitam ao professor identificar, nas disciplinas EAD ou híbridas, quais alunos apresentam um comportamento que pode levá-los ao insucesso na aprendizagem. Ao tornar explícitos os dados que encontram-se nos registros do AVA, o sistema instrumentaliza o professor para executar uma de suas tarefas principais que é o constante acompanhamento do aluno. Mais do que isso, ao fazer um tratamento gráfico da informação - agrupando dados e sinalizando com formas, cores e proporções - o software desenvolvido agiliza o processo de compreensão por parte do docente. A união desta formatação com a possibilidade de rapidamente interagir com um aluno ou com um conjunto de alunos através do próprio gráfico permite uma resposta pedagógica rápida.

Embora os resultados obtidos já indiquem a procedência da proposta, pretende-se continuar o processo investigativo com novas interações com os usuários do plugin e com um levantamento de dados mais profundo a respeito do real impacto do sistema no resgate de alunos que de outra forma teriam passado despercebidos pelo professor.

\section{Referências}

ALI, L. et al. A qualitative evaluation of evolution of a learning analytics tool. Computers \& Education, Elsevier, v. 58, n. 1, p. 470-489, 2012.

BARAB, S.; SQUIRE, K. Design-based research: Putting a stake in the ground. The journal of the learning sciences, Taylor \& Francis, v. 13, n. 1, p. 1-14, 2004.

BROOKS, C.; GREER, J.; GUTWIN, C. The data-assisted approach to building intelligent technology-enhanced learning environments. In: LARUSSON, J. A.; WHITE, B. (Ed.). Learning Analytics From Research to Practice. [S.1.]: Springer, 2014. cap. 7, p. 123-156. 
DIMOPOULOS, I. et al. Using Learning Analytics in Moodle for assessing students performance. In: . Sousse: 2nd Moodle Research Conference, 2013. p. 41.

FARIA, S. M. S. M. L. de. Educational data mining e learning analytics na melhoria do ensino online. In: . Lisboa: UNIVERSIDADE ABERTA DEPARTAMENTO de CIÊNCIAS e TECNOLOGIA, 2014.

KONRATH, M. L. P. Competências: desafios para alunos, tutores e professores da ead. In: . Porto Alegre: CINTED-UFRG, 2009.

LIMA, D. da C. B. P. Desenvolvimento, aprimoramento e consolidação de uma educação nacional de qualidade. In: . Brasília: Ministério da Educação - MEC, 2014.

LONG, P.; SIEMENS, G. Penetrating the fog: analytics in learning and education. In: Educause Review Online. [S.1.]: Publishing Press, 2011. p. 31-40.

MAZZA, R.; DIMITROVA, V. Coursevis: A graphical student monitoring tool for supporting instructors in web-based distance courses. International Journal of Human-Computer Studies, Elsevier, v. 65, n. 2, p. 125-139, 2007.

MEC. Definição de EAD. 2014. Disponível em:〈http://portal.mec.gov.br/index.php? option=com_content\&id=12823:o-que-e-educacao-a-distancia\&Itemid=230 $\rangle$.

MEC. Referenciais de Qualidade para Educação Superior a Distância. 2014. Disponível em: 〈http://portal.mec.gov.br/seed/arquivos/pdf/legislacao/refead1.pdf $\rangle$.

MOODLE.ORG. Moodle logs. 2014. Disponível em:〈https://docs.moodle.org/28/en/ Logs $\rangle$.

PARDO, A. Designing learning analytics experiences. In: LARUSSON, J. A.; WHITE, B. (Ed.). Learning Analytics From Research to Practice. [S.1.]: Springer, 2014. cap. 2, p. 15-38.

RETALIS, S. Towards networked learning analytics. In: . [S.1.]: In Proceedings of the fifth international conference on networked learning, 2006.

ROMERO, C. Educational data mining: A review of the state-of-the-art. In: . [S.1.]: IEEE - Institute of Electrical and Electronics Engineers, 2010.

SHUN, S. B. Learning analytics. In: . Moscow: UNESCO Institute for Information Technologies in Education, 2012.

SIEMENS, G.; BAKER, R. S. J. Learning analytics and educational data mining: Towards communication and collaboration. In: . New York: Columbia University, 2012.

SILVA, C. G. Ambiente virtual de aprendizagem: comunicação, interação e afetividade na ead. In: . Brasília: Revista Aprendizagem em EAD, 2012.

WANG, F.; HANNAFIN, M. J. Design-based research and technology-enhanced learning environments. Educational technology research and development, Springer, v. 53, n. 4, p. 5-23, 2005. 